\title{
Modified culture assay to obtain a diversity of hyphal structures of Ceratobasidium theobromae-VSD pathogen on cocoa
}

\author{
MUHAMMAD JUNAID ${ }^{1, \boldsymbol{v}}$, DAVID GUEST ${ }^{2}$ \\ ${ }^{1}$ Cocoa Research Group, Faculty of Agriculture, Universitas Hasanuddin. Jl. Perintis Kemerdekaan Km. 10, Tamalanrea, Makassar 90245, South \\ Sulawesi, Indonesia. Tel.: +62-411-587064, •email: muhammad.junaid@agri.unhas.ac.id; david.guest@ sydney.edu.au \\ ${ }^{2}$ Sydney Institute of Agriculture, School of Life and Environmental Sciences, Faculty of Science, The University of Sydney. 2006 Camperdown, NSW, \\ Australia
}

Manuscript received: 4 January 2021. Revision accepted: 22 March 2021

\begin{abstract}
Junaid M, Guest D. 2021. Modified culture assay to obtain a diversity of hyphal structures of Ceratobasidium theobromae, VSD pathogen on cocoa. Biodiversitas 22: 1879-1886. Ceratobasidium theobromae, a causal agent of vascular streak dieback (VSD) disease, is a fastidious Basidiomycete fungus devastating cocoa in Southeast Asia Melanesia. One sought-after in vitro technique is pathogen subculture. As an obligate parasite, growing the pathogen onto an artificial medium is always problematic. In this paper, a new design is demonstrated to obtain a single colony of the pathogen with hyphal diversity. Putative $C$. theobromae is obtained from infected leaves and petioles of cocoa using rigorous surface sterilization before culturing on standard water agar (WA) medium. Once the fungus grows out from the tissue, the infected plant tissue is then removed carefully, leaving mycelium's uninterrupted growth. About $200 \mathrm{~mL}$ of liquid Corticium culture medium (CCM) is injected to submerge the solid medium layer, nurture hyphae development, and allow generative structure formation. Molecular amplification with a pair primer of ITS 1B (forward) and 4B (reverse) specific for fungal Basidiomycete DNA with a touchdown undertook thermal cycler program. PCR product amplification successfully confirmed the presence of C. theobromae DNA collected from the samples with VSD symptomatic lesions and identified a diversity of hyphal and branching formations.
\end{abstract}

Keywords: Ceratobasidium theobromae, diverse filamentous structures, modified culture assay, PCR check

\section{INTRODUCTION}

The VSD pathogen on cocoa causes branch dieback, streaking cambium followed by chlorotic and necrotic lesions of the leaf, spotted axillary buds and cracked veins and midrib of leaves even immature tree death (Keane and Prior 1991; Marelli et al. 2019; Samuels et al. 2012). Infection of vascular tissues can cause the imbalance of nutrients and water flow in host systems (Agrios 2012), causing specific leaf lesions. The infection can also cause changes in plant hormone and secondary metabolites (McMahon and Purwantara 2016; Santoso et al. 2017), impacting the symptomatic lesions. The initial symptom is characterized by interveinal leaf chlorotic and necrotic lesions or general chlorosis and leaf loss impairing photosynthetic functions. Swollen lenticels on the bark and darkly stained vascular traces are another sign. Under wet conditions, reproductive basidiocarps form on leaf scars or cracks in the leaf midrib and veins, generating an inoculum source as the wind transmits basidiospores to new hosts (Keane 1981). The causal agent of VSD is a Cantharellales basidiomycete described by Talbot and Keane (1971) and given the name Oncobasidium theobromae. Along with applying molecular biology techniques, Samuels et al. (2012) proposed the revision of this nomenclature to Ceratobasidium theobromae.
VSD symptoms vary widely in Sulawesi region. For new symptomatic patterns, an earlier necrotic without chlorotic leaf lesions naturally occurs, but late death of cells after chlorosis as an old symptom is quite frequent in the field (Junaid 2018). Previously, the distribution of different symptoms was patchy (McMahon and Purwantara 2016), but most recently, the symptom has spread elsewhere in Sulawesi Region (Junaid 2018; Junaid et al. 2020).

Growing putative $C$. theobromae in vitro to obtain its generative structure is challenging (Guest and Keane 2007). Almost the past 40 years after the successful experiment of growing Oncobasidium theobromae on Corticium culture medium (CCM) to obtain basidiospores was reported (Lam et al. 1988), but no further report of a repeated successful attempt to attain generative structure has been achieved. Although the pathogen's known genome was successfully conducted (Ali et al. 2019) through plant DNA extraction protocol (Samuels et al. 2012), the genome obtained from subcultured mycelium extraction is still unclear. Since then, an effective method of in vitro assay technique to obtain generative structure has been focused. This paper will demonstrate a modified in vitro method to collect basidiomata-like and a single pure colony of the fastidious Basidiomycete fungus. 


\section{MATERIALS AND METHODS}

\section{Sample collection and microscope check for the presence of pathogen}

Before doing the initial in vitro culture, hands and tools must be washed with $70 \%$ ethanol or sanitizer gel. The sample of interest was usually an infected petiole or a midrib because the tissue was soft and easy to separate during treatment. The leaf sample was kept in water and airproof to maintain moisture inner plastic bag. The tip of the plastic bag was wrinkled to ensure no air leaking. In in vitro assay, the branch is separated among leaves. To ensure the presence of $C$. theobromae hyphae on the tissue, a microscopic check is underlined as follows: At the beginning of in vitro activity, following a Koch Postulate as standard procedure to prove the cause of the disease, a check of fresh infected tissue is essentially conducted to ensure pathogen of VSD disease symptom inside the tissue of vascular cambium. Suppose the VSD pathogen's presence on vascular tissue is indicated. In that case, initial isolation and culture work, hyphae elongate in the vascular cambium with branching Rhizoctonia-like pattern, which most probably is in the group of Basidiomycete species viz. C. theobromae (Figure 1, read arrowhead).

\section{Surface sterilization, isolation and culture}

In the entire isolation activity, as the initial stage, surface sterilization is an important part. Surface sterilization can enable a single pure colony to be obtained in the further step. In the lab activity, an infected petiole is cut longitudinally $3-5 \mathrm{~cm}$ long with a sterilized surgery blade and transferred into $70 \%$ Ethanol, $2.5 \%$ Clorox and twice submersion of sterilized water respectively, for 3-5 minutes and dried. A bottle of Clorox as a whitener or disinfectant purpose can be bought in local trade stores, and a $15 \mathrm{~mm}$ petri dish, sterilized blade and $70 \%$ Ethanol are easily found in the local Pharmacy store. Modification of surface sterilization depends on the thickness or softness of tissue. All activity is under sterilization condition on the cabinet using a flame. Before the sample is transferred into WA, the infected petiole or midrib is gently dried on sterilized blotting paper. Isolating wet tissue of interest on culture and subculture media risks contamination of 'fungal pirate' (including endophytes) inhabiting culture medium side by side with hyphal $C$. theobromae.

To maintain the pathogen, $15 \%(\mathrm{~g} / \mathrm{mL})$ water agar (WA) is prepared, consisting of $15 \mathrm{mg}$ agarose mixed with $100 \mathrm{~mL}$ distilled water. The final WA solution is stirred automatically until the solution boils. A $5 \mathrm{mg}$ tablet of streptomycin is dissolved into agar solution $(5 \mathrm{mg} / 1000 \mathrm{~mL}$ solution) once the temperature medium drops at about 40 ${ }^{\circ} \mathrm{C}$. Soon after added with an antibiotic, $100 \mathrm{~mL}$ warm agar is poured into a $15 \mathrm{~mm}$ disposable petri dish. The lid is kept open until agar cools and crystallizes. A disposable transparent petri dish is highly recommended for frequent histological assessment purposes. Glass petri dish does not fit under the lens of a microscope and blurred images result.

In the subculture, a modified Corticium culture medium (CCM) (Leonian 1924) is recommended as its nutrient composition to fit for basidiomycete, including Rhizoctonia species. Liquid CCM is prepared by dissolving $1.25 \mathrm{mg}$ potassium dihydrogen phosphate $\left(\mathrm{KH}_{2} \mathrm{PO}_{4}\right), 0.59 \mathrm{mg}$ Magnesium sulfate $\left(\mathrm{Mg}_{2} \mathrm{SO}_{4} 7 \mathrm{H}_{2} \mathrm{O}\right), 6.25 \mathrm{mg}$ Maltose, $0.625 \mathrm{mg}$ peptone and $6.25 \mathrm{mg}$ malt extract in $1000 \mathrm{~mL}$ distilled water. The reagents are mixed with a magnetic stirrer, boiled and autoclaved for 15 minutes at $121^{\circ} \mathrm{C}$. The solution, separately, is cooled down and kept under sterilized conditions. Infected petiole that is fully treated with a set of surface sterilization is transferred carefully into a $15 \mathrm{~mm}$ disposable petri dish with a sterilized blade and laid in the central agar medium. Daily observation is recommended to aware of endophytic interferences. In this section, a daily assessment under the microscope is essential to discriminate whether hyphae belong to $C$. theobromae or endophytes.

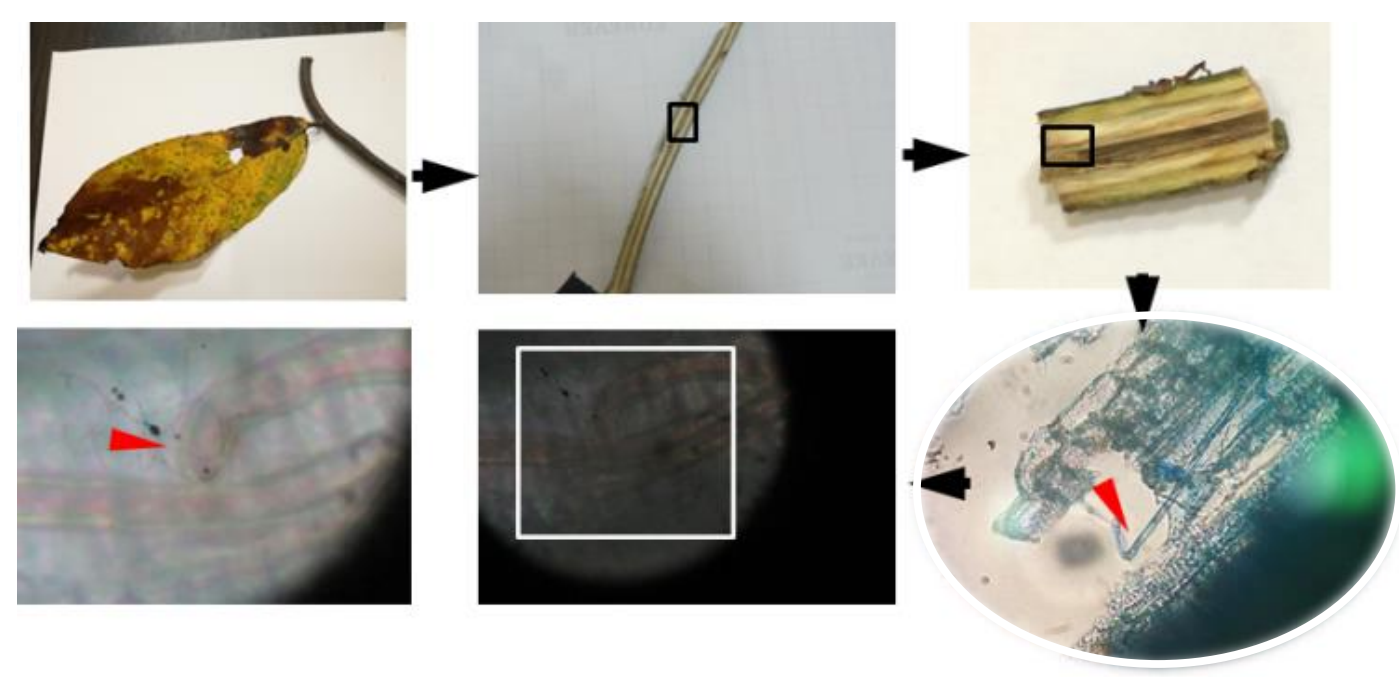

Figure 1. An initial check of VSD symptom confirms the presence of the pathogen through microscopic analysis 


\section{Mycelium separation and fungal DNA extraction}

For the next stage, the separation between mycelium and solid agar medium is undertaken by melting agar method (Figure 2.A), since the highest risk of contamination in subculture occurs. Thus, collecting mycelium from the original agar medium seems to be a much more effective way. By melting agar medium at low heat (pers. comm. with Agus Purwantara), mycelium and melted agar is a part. In this recent experiment, to optimize the output, improving the isolate separation technique is essential by adding a metal screen in the lower solid agar medium. Agar medium that is heated about $70^{\circ} \mathrm{C}$ will dissolve. The increase of temperature instrument is incrementally set, keeping in mind that overheated agar medium can destroy the sample. After that, to remove residual agar, the filaments are washed in sterile water gently several times. So, once the filaments float and water bubbles initiate to rise, the screen is lifted, moved and rinsed into the second and the third glass petri dish, respectively, before capturing with forceps for fungal DNA extraction purpose. For fungal DNA extraction, utilizing lysis buffer, a commercial kit can be used (https://www.bioline.com/au/isolate-iiplant-dna-kit.html).

\section{Testing putative Ceratobasidium theobromae fragment with PCR amplification}

PCR amplification aims to confirm the presence of $C$. theobromae mycelium grown by the previous modified isolation technique. Mycelium obtained from a modified culture in vitro is extracted using a commercial buffer kit and then extracted DNA tested by PCR amplification of the ITS region (White et al. 1990). For Basidiomycete species, utilizing a pair of primers of ITS 1B (forward) and ITS 4B (reverse) is a robust tool to discriminate among other fungi and Basidiomycete species in the tissue (White et al. 1990). This pair of primers (ITS $1 \mathrm{~B}$ and $4 \mathrm{~B}$ ) amplifies the 5,8S ITS region for Basidiomycete species. For primer Than
ITS1 and Than ITS2, fungal $C$. theobromae nucleotide is specifically amplified (Samuels et al. 2012). However, in this experiment, the primers ITS 1B and 4B were applied, and the confirmation of the culture as a Basidiomycete combined with microscopic observation enabled the conclusion that the culture was most likely to be $C$. theobromae.

Figure 2.B shows fragments of DNA amplification with a pair of primers for basidiomycete species to obtain fungal DNA amplification. Thermal cycler was set as the following touch down PCR reported by (Samuels et al. 2012). Touchdown PCR is strongly preferred for its high specificity, robustness, and downstream PCR sensitivity that can influence sequence data (Korbie and Mattick 2008; $\mathrm{Wu}$ et al. 2005). Touchdown PCR consists of $2 \mathrm{~min}$ at $94^{\circ} \mathrm{C}$ pre-denaturation, followed by initial 15 cycles of PCR amplification. The annealing temperature in the first amplification cycle is $65^{\circ} \mathrm{C}$, which is subsequently incrementally reduced by $1^{\circ} \mathrm{C}$ per cycle over the subsequent 15 cycles. Then an additional 35 cycles are performed, each consisting of $30 \mathrm{~s}$ denaturation at $94^{\circ} \mathrm{C}$, a $30 \mathrm{~s}$ annealing at $48^{\circ} \mathrm{C}$, and a $1 \mathrm{~min}$ extension at $72^{\circ} \mathrm{C}$ concluding with a $10 \mathrm{~min}$ extension at $72^{\circ} \mathrm{C} .1 \%$ agarose to amplify PCR products (amplicons) is stained with ethidium bromide.

\section{RESULTS AND DISCUSSION}

\section{Isolation and culture}

The growth of filamentous $C$. theobromae initiates to form straight hyaline hyphae with the presence of flanking compartments (septa) in every emerging new branching structure and near right-angle branching pattern with a septum on the bottom and somewhat yellowish mycelium (Figure 3).

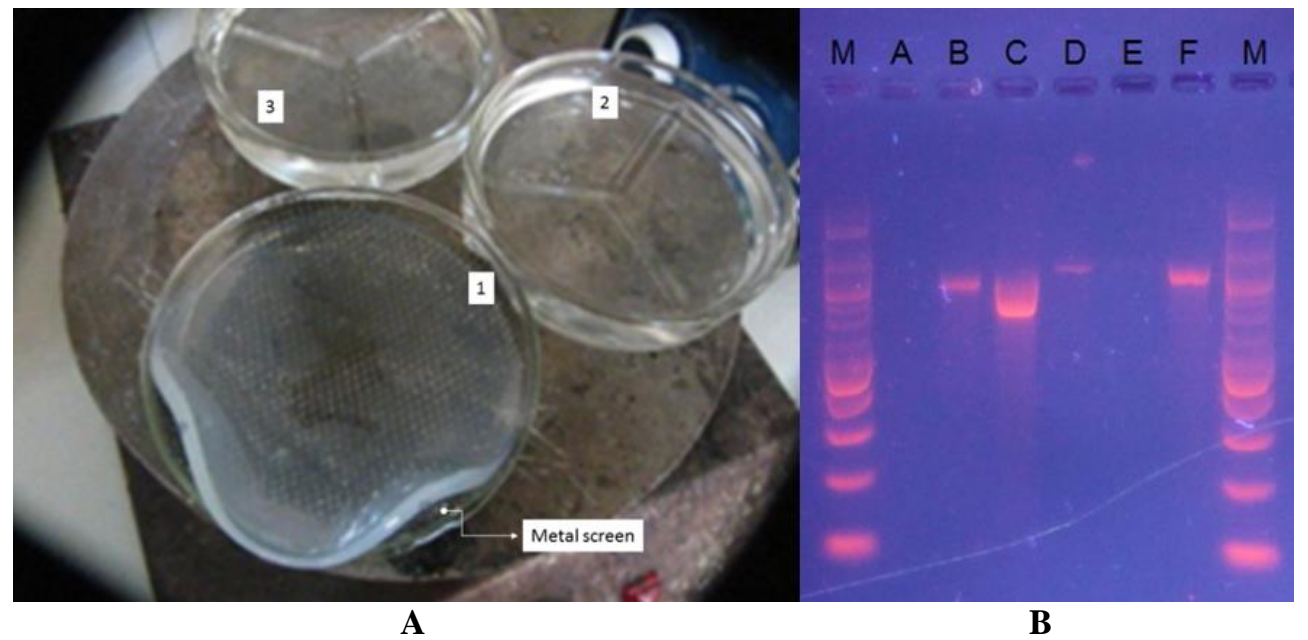

Figure 2. A. A simple separation technique between filaments of interest and solid agar medium was performed. Underneath the agar with mycelium was a metal screen to filter in dissolving agar and in separating Ceratobasidium theobromae filaments. Once agar was melted, the metal screen was lifted and moved to a new glass petri dish with clean water subsequently. B. Gel electrophoresis check was utilized to test a DNA sample with PCR amplification obtained from the melting agar technique. The original mycelium was isolated from infected leaf tissue indicating VSD symptoms. $\mathrm{M}=$ marker (100 bp); sample A was negative control (only PCR mixture without DNA template) B, C, D, E, and F were sample DNA templates in primer (ITS 1B and 4B) with 1-, 10- and 100-times dilutions and E= Ascomycete fungus. The presence of DNA fragments of interest performs on the gel agarose 


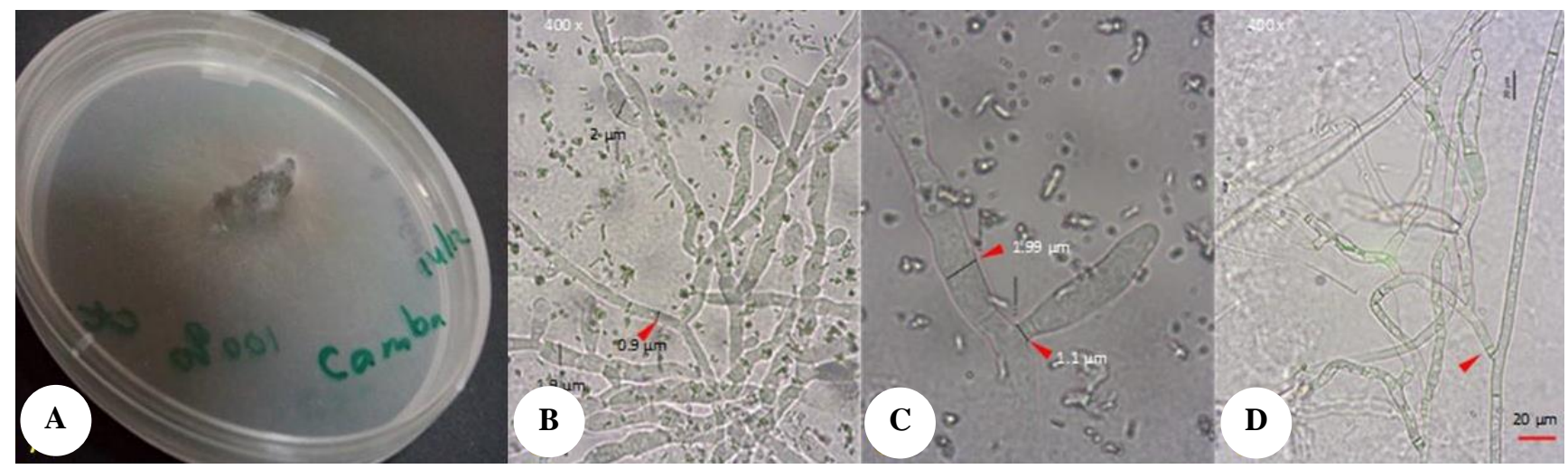

Figure 3. Development of filamentous VSD pathogen on the original WA medium on a $15 \mathrm{~mm}$ disposable petri dish is rather yellow with low density hyphae (A). Hyphal wall ranges from $0.9 \mu \mathrm{m}$ to $2 \mu \mathrm{m}$; a right angle (red arrowhead-B, C, D) of new emerging hyphal branching has septum (arrowhead-D) and is inflated (C); and main hypha is straight (D). Aerial hyphae frequently develop on the agar medium. In WA agar medium, poor generative structure and branching pattern formed. Average width of hyphae is approximately $5 \mu \mathrm{m}$

In contrast to hyphal growth on the origin WA medium, hyphae, and branching structure vary to form in modified culture technique (Figure 4). Diversity of hyphal and branching patterns is obtained from secondary transfer to WA, PDA, and CCM media. Aerial generative mycelium forms to spread on the lid. Aerial mycelium formation is closely associated with $C$. theobromae (Samuels et al. 2012), often spread on the lid but not on the agar layer. The diverse basidia structures develop in the hyphal apex, which usually becomes nuclear fusion and meiosis. In these pictures, basidiomata and protobasidia are examined, similar to a group of Thanatephorus (Stalpers and Andersen 1996). In this experiment, proto-sterigmata are absent.

Regarding the Ceratobasidiales and Tulasnellaceae as a group of Rhizoctonia species, typically basidium forms vary. The hypha has monilioid cell formation, and the right-angle branching structure is generated from the primary hyphae (Figure 4.D-E). It is slightly constricted at the origin of branching with the presence of a septum. In the main hypha, every cell contains multinucleate nuclei (Figure 4.A), and septum (Figure 4.D-E) develops: in between septa represents one cell. Protobasidia form in a pair of basidium. This evidences that in this method, hypha fusion occurs (Figure 4.I). A set of hyphae is nuclei less connecting with multi nuclei hypha. Unlikely, poor generative structures form was found in a classical way (Figure 3 ).

In Figure 4, all cultures originated from cocoa hillside village in Camba, Maros South Sulawesi, where VSD pathogen was never reported until the study was conducted. Putative $C$. theobromae isolate is transferred from the original WA medium to PDA and CCM media with special handling (Figure 6). Various generative mycelium patterns form to obtain. In contrast to Figure 4, with a classical culture technique shown in Figure 3, hyphal structure and branching pattern are less diverse, and no proto-basidium and basidiomata were generated.

Also from Figure 4, it is noted that aerial generative mycelium that spread on the lid is examined under the microscope. There is a formation of basidia structures on the various hyphal apex, which are usually nuclear fusion and meiosis. Diverse basidiomycete hyphal forms are significantly obtained with a modified subculture medium.
A rare hyphal structure formation such as basidiomata and protobasidia develops using the common culture technique. (A) $\mathrm{Bi}^{-}$and multi-nuclei present in the hyphal compartment. (B) Aerial hyphae emerge and spread underneath the lid. (C) Double-laminate basal hyphae form (black arrow), which has become a distinctive feature on each division. (D) Emerging secondary hyphae and branching patterns form to have typical compound sympodia structure. (E) The presence of a septum near the point of origin is a distinctive feature in a group of fungal Rhizoctonia (Murray 1984), which belongs explicitly to Basidiomycete (Roberts 1999). Bent branching hypha can occur, but it is not very often. (F) Protobasidia emerges from a particular type of umbellate sympodium structure obtained from secondary hypha. (G) A pair of basidium forms on the hyphal branching apex (arrowhead). (H) Inflated hyphae emerge and become basal basidium which forms a type of simple sympodium structure. (I) A set of hyphal fusion connects nuclei-free and multi-nuclei (arrowhead) within septation.

\section{Mycelium separation and fungal DNA extraction}

$C$. theobromae behavior on the agar medium is slow to grow and usually shapes aerial filaments, while filamentous endophyte community is uncommon. Regarding aerial filaments, an attempt to open a $15 \mathrm{~mm}$ petri dish is not recommended since $C$. theobromae filaments spread on the bottom lid and will destroy structures. Therefore, injecting the CCM medium into the transparent lid of the petri dish could accelerate the form of generative structures. A sterilized needle of $100 \mathrm{~mL}$ syringe is a standard aseptic tool that could be familiarly found in a local pharmacy store to feed $C$. theobromae filaments with CCM medium. Injecting $200 \mathrm{~mL}$ CCM into WA through penetrating needle into the upper lid significantly achieves less contaminating risk. Due to a fastidious pathogen, the needle is initially heated for easy penetration purposes and then punctured gently toward the lid. Once the needle tip is inside the petri dish, the CCM medium is injected gently. The needle hole remaining is locked using sterilized parafilm. A $15 \mathrm{~mm}$ petri dish with WA and CCM media returned to $25^{\circ} \mathrm{C}$ incubators with a rotating $30 \mathrm{rpm}$ shake. 

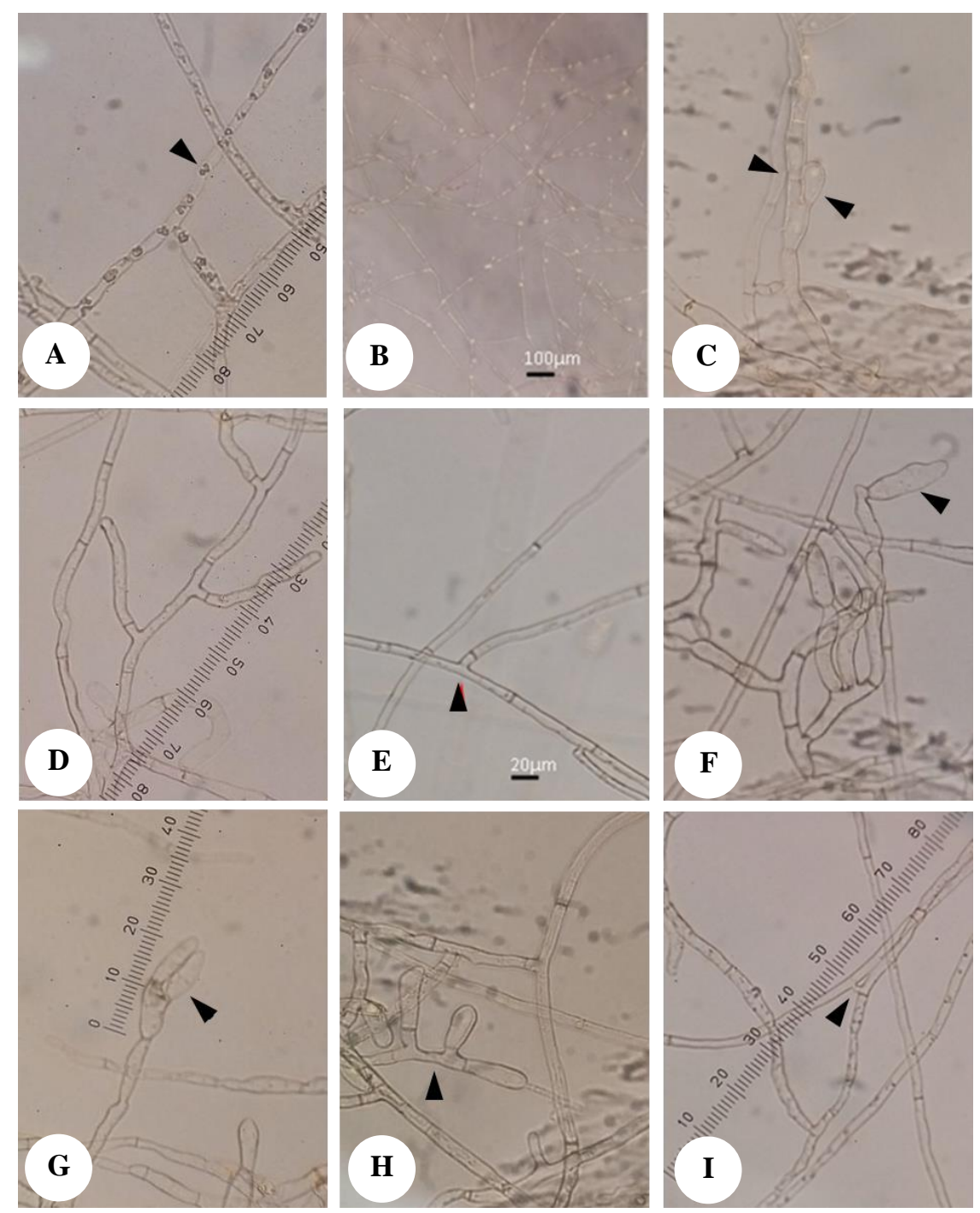

Figure 4. Diversity of hyphal and right-angle branching structure formations (arrows) are obviously obtained from modified culture medium

\section{Problems of subculturing an obligate parasite in vitro}

Most likely, growing a single colony of fungal Ceratobasidium theobromae in vitro is that another fungal endophyte is the most significant potential problem. Although fungal isolation is handled carefully and rigorous initial surface sterilization to avoid a living microbe in the outer surface layer of the tissue, an endophytic community still keeps on growing out from the tissue.

In Figure 5.A, frequent fungal contaminant grows (black circle) side by side with $C$. theobromae (white circle) on the medium. Figure 5.B, emerging mycelium opposite side occurs between $C$. theobromae $(\checkmark)$ and contaminant (x) in the infected petiole. Figure 5.C, a curly main hypha (black arrowhead) is a typical fungal contaminant, while (red arrowhead) main hypha of $C$. theobromae is straight. Hyphal structure and septation near right-angle branching pattern is the main key determination to distinguish between Basidiomycete species (e.g. $C$. theobromae) and fungal Ascomycete species as endophytes.
Since an attempt to obtain a single pure culture of $C$. theobromae is challenging due to the presence of fungal endophyte (Figure 5), a rapid separation between hyphae of interest and endophyte is necessary before endophyte initiates to colonize the medium. Some endophytes can grow very fast once it grows out from the infected source. However, this study shows the VSD pathogen to grow earlier out from the tissue source and slower on culture than other fungi. Unlikely previously reported, maintaining pure $C$. theobromae mycelium on CCM medium is not an easy task. At first, growth on WA medium reveals a branching pattern that is Rhizoctonia-like but once transferred, and the mycelium is overgrown by other fungi (most likely endophytes). Therefore, repeated transfer of hyphae to a new solid medium can result in a single colony and avoid contamination from the endophytic community. Aerial filaments can be comfortably observed onto the lid of a disposable petri dish (Figure 4.B). Transfer of a small portion on the new agar medium can help the filaments to spread to the lid as aerial hyphae. Aerial hyphae can also be 
used to distinguish between $C$. theobromae and fungal endophytes.

In initial growth, normally $C$. theobromae filaments grow out earlier from the source of infected tissue than another fungus, but once the contaminant grows, it colonizes the medium. From day 2 to day three after isolation, $C$. theobromae filaments frequently grow out from the source of infected tissue. Therefore, modified in vitro technique with speed removal of infected tissue and hyphae of interest remaining is essential. The lid of the petri dish is wrapped up and moved to an incubator at $25 \mathrm{C}$ temperatures. If $C$. theobromae filaments, however, grow out opposite from endophytic filaments on surface WA medium, a tiny portion of agar medium with $C$. theobromae filaments is cut and rapidly transferred into a new WA medium. Mixing between $C$. theobromae and contaminant hyphae makes separation impossible to conduct. The growth of filamentous $C$. theobromae initiates to form straight hyaline hyphae, the presence of flanking compartments (septa) in ever-emerging new branching, near right-angle branching pattern with a septum on the bottom and somewhat yellowish mycelium (Figure 3).

Several recommendations to obtain a single pure colony with diverse generative structure through a modified isolation technique in vitro are proposed. Firstly, in the surface sterilization and isolation stage, hand sterilization and aseptic lab equipment are essential to support a single pure $C$. theobromae colony on media. Liquid disinfectants such as Ethanol and Clorox are only used at once during isolation. Figure 5 describes an entire modified in vitro activity as follows.

Secondly, at the beginning of in vitro activity, following a Koch Postulate as standard procedure to prove the disease's cause, check and recheck of fresh infected tissue is essentially conducted to ensure pathogen of VSD disease symptom inside the tissue of vascular cambium. The activity confirms the presence of $C$. theobromae hyphae in the culture.
The primary focus is an interference of the pathogen of VSD by contaminants. Due to potential contamination in every single hyphal transfer, earlier segregation between hyphae of interest and infected tissue on the solid agar medium and fungal contaminant is another essential consideration. Once the VSD pathogen's presence on vascular tissue is indicated, initial isolation and culture commence. If hyphae elongate in the vascular cambium with a branching Rhizoctonia-like pattern, it suggests a group of Basidiomycete species representing by $C$. theobromae (Figure 1- red arrowhead). In Figure 6, the diagram based on the experiment shows how to obtain a pure culture of $C$. theobromae by reducing contamination risk. In this section, the protocol's essential part is to check before surface sterilization and after isolating hyphae on the culture medium. Recheck continues once hyphae of interest grow before injection section is conducted.

Besides, subculture injection through the lid of the plastic petri dish is put forward. Subculture medium rich in nutrients helps in accelerating generative structure formation. Since the contamination and mycelium disturbance by repeated lid open and close of petri dish often occurs, the subculture medium injection is a better way. In this modified technique, hyphal transfer to a new subculture medium is not recommended; otherwise, the contaminant, fungal 'pirate'-like, grows earlier. They grow side by side with hyphal $C$. theobromae before they colonize the entire agar surface.

Furthermore, the quick separation between hyphae of interest and another fungus in earlier growth is suggested. Commonly, $C$. theobromae filaments grow out earlier from the source of infected tissue than endophytes. In between day 2 and day 3 after isolation, $C$. theobromae filaments frequently grow out from the start of infected tissue. However, once another fungus associated with VSD symptom grows, they colonize the medium rapidly. Therefore, in the modified in vitro technique, speed removal of infected tissue and hyphae of interest remains.

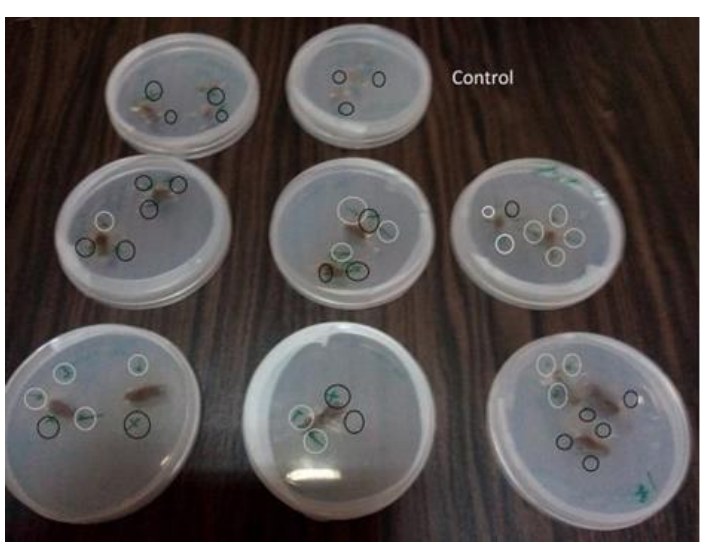

A

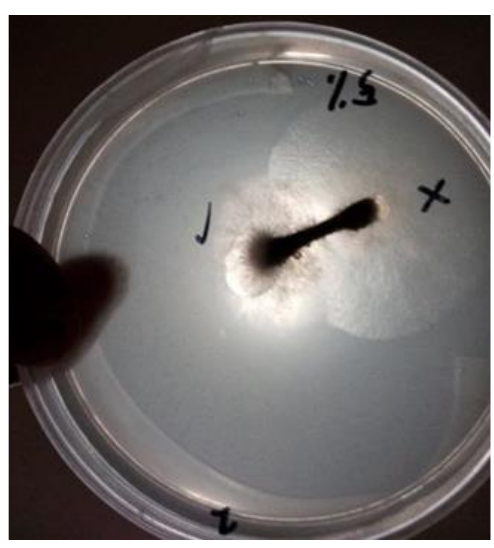

B

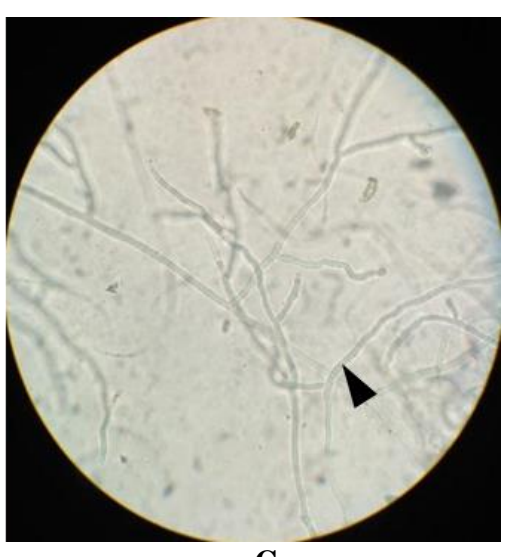

C

Figure 5. Isolation of fungal association obtained from infected tissue on WA medium 


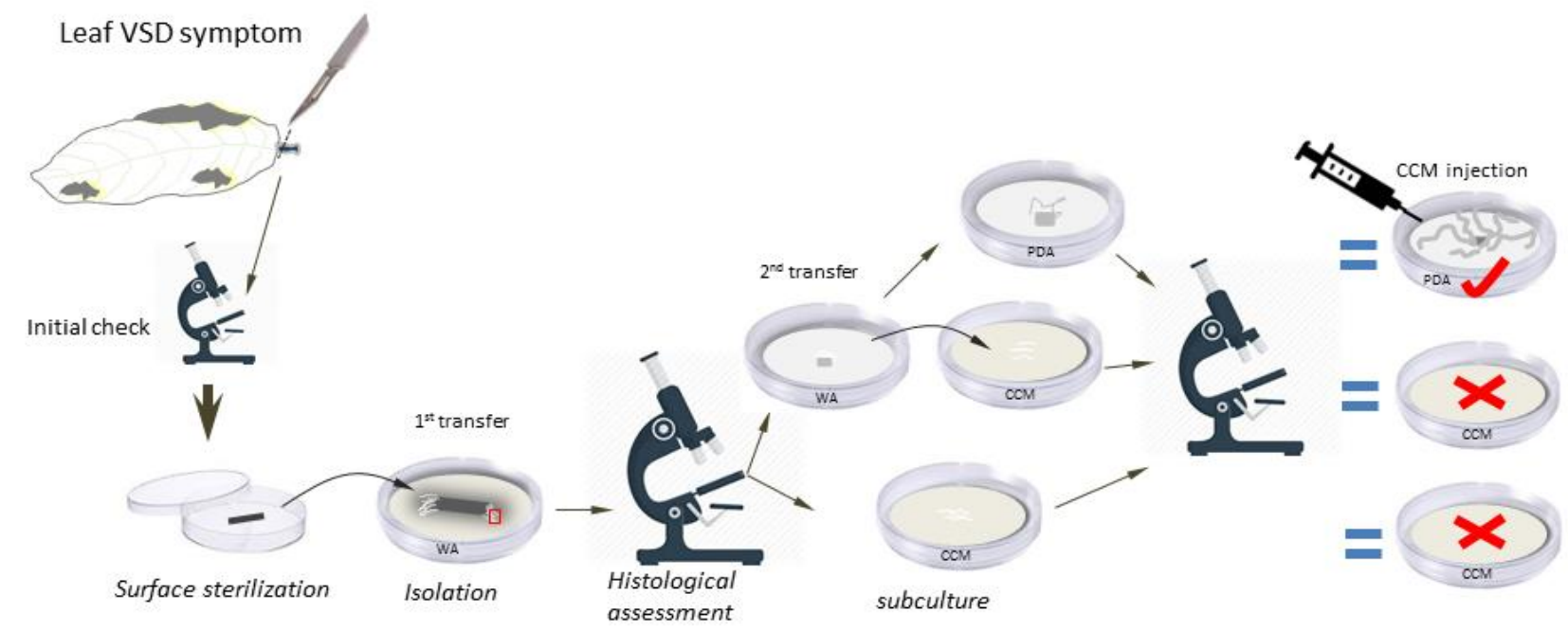

Figure 6. An illustration of modified protocol to obtain a pure culture of an obligate parasite. By integration between culture and subculture, a diversity of hyphal structures of C. theobromae is produced (Junaid 2018).

In many cases, $C$. theobromae filaments grow out opposite from endophytic filaments on surface WA medium and hence a tiny portion of agar medium with $C$. theobromae filaments is cut and transferred into a new WA medium. The lid of the petri dish is wrapped up and moved to an incubator at $25^{\circ} \mathrm{C}$ temperatures. The hyphal character of $C$. theobromae as previously described above.

The melting agar technique is also utilized to avoid the risk of DNA contamination since subculturing an obligate parasite becomes a significant issue that causes to fail in achieving crude DNA of interest. Harvesting mycelium from melting agar is a simple way and reduces the potential risk of contamination and time-consuming. This technique can also provide sufficient mycelium weight for fungal DNA isolation and extraction, as mycelium can be entirely harvested in the solid medium.

Eventually, to rectify fungal hyphae of interest cultured in the modified Invitro protocol, molecular PCR amplification is robust. Using primers for Basidiomycete species is sufficient and allows only DNA of Basidiomycete species to be amplified while fungal pirate DNA is absent. The only known fungal Basidiomycete pathogen causing VSD disease on cocoa is $C$. theobromae. In this experiment, DNA fragments of samples obtained from in vitro modified technique have amplified with primers for Basidiomycete species, and no band is in control. The finding suggests that hyphal shapes, new branching patterns and generative structures previously obtained from modified in vitro technique belong to $C$. theobromae instead of fungal contaminant.

In conclusion, attempting to characterize VSD disease pathogen in culture and subculture media faces a significant issue as the endophyte community acting like a pirate is more dominant. There is a lack of literature on achieving diverse basidiomata or protobasidia on the agar medium. However, the current technique has successfully obtained the diversity of hyphal branching patterns and generative structures. We inferred that with the current protocol, diverse generative structures of $C$. theobromae are successfully received, while in WA medium, a lack of hyphal generative structure form. The present method can support in understanding of $C$. theobromae biology and the original host in the future.

\section{ACKNOWLEDGMENTS}

We acknowledge Peter McMahon for field visit in Camba Village and Agus Purwantara for technical advice. We also thank heads of Forest Biotechnology Lab., Faculty of Forestry, as well as Molecular and Plant Propagation Lab., Faculty of Agriculture, Hasanuddin University, Makassar, Indonesia for facility support for invitro assay and PCR work

\section{REFERENCES}

Agrios G. 2012. Plant Pathology. Burlington: Elsevier Science.

Ali SS, Asman A, Shao J, Firmansyah AP, Susilo AW, Rosmana A, McMahon P, Junaid M, Guest D, Tee YK, Meinhardt LW, Bailey BA. 2019. Draft genome sequence of fastidious pathogen Ceratobasidium theobromae, which causes vascular-streak dieback in Theobroma cacao. Fungal Biol Biotechnol 6 (1): 14. DOI: 10.1186/s40694-019-0077-6.

Guest D, Keane P. 2007. Vascular-Streak Dieback: A New Encounter Disease of Cacao in Papua New Guinea and Southeast Asia Caused by the Obligate Basidiomycete Oncobasidium theobromae. Phytopathology 97 (12): 1654-1657. DOI: 10.1094/PHYTO-97-121654.

Junaid M. 2018. Geographic diversity of "new" and "old" symptom of vascular streak dieback disease on cocoa, caused by Ceratobasidium theobromae in Sulawesi. [Dissertation]. The University of Sydney, Sydney, NSW.

Junaid M, Purwantara A, Guest D. 2020. First report of vascular streak dieback symptom of cocoa caused by Ceratobasidium theobromae in Barru District, South Sulawesi. IOP Conf Ser Earth Environ Sci 486: 012170. DOI: 10.1088/1755-1315/486/1/012170. 
Junaid M, Samuels GJ, Ismaiel A, Rosmana A. 2009. Detection of Thanatephorus theobromae, Cause of Vascular Streak Dieback Disease of Cocoa in Cocoa Tissues Luwu Regency, South Sulawesi. International Cocoa Pest and Disease Conference, Bali, Indonesia.

Keane PJ. 1981. Epidemiology of vascular-streak dieback of cocoa. Ann Appl Biol 98 (22): 227-241. DOI: 10.1111/j.17447348.1981.tb00756.x.

Keane PJ. 1992. Diseases and Pests of Cocoa: An Overview. Cocoa Pest and Disease Management in Southeast Asia and Australasia, Rome.

Keane P. 2013. Vascular Streak Dieback of Cocoa: Recent Changes in Symptoms and Prospects for Control. Paper presented at the Celebes International Seminar Cocoa, Makassar.

Korbie DJ, Mattick JS. 2008. Touchdown PCR for increased specificity and sensitivity in PCR amplification. Nature Protocols 3 (9): 1452 1456. DOI: $10.1038 /$ nprot.2008.133.

Lam CH, Varghese G, Abidin MAZ. 1988. In Vitro Production of Oncobasidium theobromae Basidiosphores. Trans Br Mycol Soc 90 (30): 505-507. DOI: 10.1016/S0007-1536(88)80168-2

Leonian LH. 1924. A study of factors promoting pycnidium formation in some Sphaeropsidales. Am J Bot 11 (1): 11-50. DOI: $10.2307 / 2446570$

Marelli JP, Guest DI, Bailey BA, Evans HC, Brown JK, Junaid M, Puig AS. 2019. Chocolate Under Threat from Old and New Cacao Diseases. Phytopathology 109 (8): 1331-1343. DOI: 10.1094/phyto12-18-0477-rvw.

McMahon P, Purwantara A. 2016. Vascular Streak Dieback (Ceratobasidium theobromae): History and Biology. In: Bailey AB Meinhardt WL (eds.), Cacao Diseases: A History of Old Enemies and New Encounters. Springer International Publishing, Cham, Switzerland.
Murray DL. 1982. A Modified Procedure for Fruiting RHIZOCTONIA SOLANI on Agar. Trans Br Mycol Soc 79 (1): 129-135. DOI: 10.1016/S0007-1536(82)80198-8

Roberts P. 1999. Rhizoctonia-Forming Fungi. A taxonomic guide Book: Herbarium Royal Botanic Garden, Kew.

Samuels GJ, Ismaiel A, Rosmana A, Junaid M, Guest D, McMahon P, Cubeta MA. 2012. Vascular Streak Dieback of cacao in Southeast Asia and Melanesia: in planta detection of the pathogen and a new taxonomy. Fungal Biol 116 (1): 11-23. DOI: 10.1016/j.funbio.2011.07.009

Santoso TI, Miftahudin M, Sulistyaningsih YC, Wiyono S. 2017. Analysis of secondary metabolites as potential phytoalexins, their secretion sites and proposed resistance markers to vascular streak dieback in Theobroma cacao L. Pelita Perkebunan 33 (1): 10-23.

Stalpers JA, Andersen TF. 1996. A synopsis of the taxonomy of teleomorphs connected with Rhizoctonia s.l. In: Sneh B, Jabaji-Hare S, Neate S, Dijst G (eds.). Rhizoctonia Species: Taxonomy, Molecular Biology, Ecology, Pathology and Disease Control. Springer-Science Business Media, B.V., Nederland.

Talbot PHB, Keane PJ. 1971. Oncobasidium: A New Genus of Tulasnelloid Fungi. Aust J Bot 19: 203-206.

White T, Bruns T, Lee S, Taylor J. 1990. Amplification and direct sequencing of fungal ribosomal RNA genes for phylogenetics. Academic Press, San Diego, CA.

Wu WM, Tsai HJ, Pang JH, Wang HS, Hong HS, Lee YS. 2005. Touchdown thermocycling program enables a robust single nucleotide polymorphism typing method based on allele-specific realtime polymerase chain reaction. Anal Biochem 339 (2): 290-296. DOI: $10.1016 /$ j.ab.2005.01.037 